\title{
ALTERNATING PERIOD-DOUBLING CASCADES
}

\author{
PHILIP J. ASTON AND NEIL BRISTOW \\ Department of Mathematics \\ University of Surrey \\ Guildford \\ Surrey GU2 7XH \\ U.K.
}

\begin{abstract}
We consider period-doubling cascades in two-dimensional iterated maps. We define forward and backward period-doubling bifurcations, and use these concepts to describe an alternating period-doubling cascade in which forward and backward perioddoubling bifurcations alternate. By tracking the eigenvalues of a typical map throughout such cascades we show that two-dimensional maps may give rise to two qualitatively different alternating period-doubling cascades. We apply renormalisation theory to one class of alternating period-doubling cascades, and derive universal spatial scalings for such cascades from fixed points of the appropriate renormalisation operator. We also derive universal parameter scalings for these cascades from the eigenvalues of the linearisation of the renormalisation operator, and provide the corresponding eigenfunctions. The theory is illustrated by an example.
\end{abstract}

MSC Classification: 37G35, 37E20

\section{INTRODUCTION}

We consider period-doubling bifurcations, and certain cascades thereof, which can arise in iterated maps of dimension two or more. Our main aim will be to describe and analyse a period-doubling cascade with a particular geometric structure, which we term an alternating period-doubling cascade.

Period-doubling bifurcations are a well studied codimension one local bifurcation of discrete dynamical systems which arise when the Jacobian matrix of the map has an eigenvalue that passes through -1 . They are usually classified according to their criticality, or the specific relationship between the stability of the different branches of solutions in a neighbourhood of the bifurcation point, in which a supercritical bifurcation gives rise to a stable period two branch which coexists with an unstable period one branch and a subcritical bifurcation has an unstable period two branch coexisting with a stable period one branch. This concept works well for one-dimensional maps, but for higher dimensional maps, we note that there are additional eigenvalues of the Jacobian which do not change sign in the neighbourhood of a bifurcation point, and if some of these are outside the unit circle, then there are no stable solutions occuring in either type of bifurcation. Normal form calculations and conditions for super- (sub-) criticality appear widely in the literature, for example [1].

Cascades of period-doubling bifurcations have also been widely studied, in both one (e.g. $[2,3,4,5])$ and higher (e.g. $[6,5,7])$ dimensions. The classification of period-doubling bifurcations often extends to the classification of period-doubling cascades, so that one may refer to supercritical, or subcritical, period-doubling cascades. A classification of this

Date: August 6, 2013. 
type provides information about the stability properties of a period-doubling cascade, but yields little information about its geometry. In particular, knowing whether a perioddoubling bifurcation is supercritical or subcritical alone is not enough to determine the direction (with respect to the parameter) in which the bifurcating branches of solutions emerge from the bifurcation point.

In contrast to the usual approach of taking stability as the key property of interest when examining period-doubling cascades, we focus our attention on the direction in which bifurcating solutions emerge from a period-doubling bifurcation. To provide a basis for describing cascades in this way, we define forward and backward (local) period-doubling bifurcations. From these definitions we are able to describe the essential geometric property of an alternating period-doubling cascade, in which forward and backward bifurcations alternate, and this is our main object of study.

We analyse and classify alternating period-doubling cascades in two-dimensional maps by tracing the possible paths of eigenvalues as we proceed through the cascade, noting that these paths will be related to and restricted by the geometrical structure we impose on the cascade. We show that alternating period-doubling cascades cannot occur for onedimensional maps and that for two-dimensional maps, there are precisely two stability configurations that allow for such an infinite cascade.

A key tool used to analyse the asymptotic properties of period-doubling cascades is renormalisation theory. Feigenbaum $[3,4,5]$ examined the limiting behaviour of onedimensional period-doubling cascades and derived universal spatial and parameter scalings which are valid for a wide class of one-dimensional maps. A number of extensions of this original theory have been carried out, including extensions to higher dimensional maps $[7,8]$, systems of area-preserving maps $[9,10]$, infinite cascades of period $p$-tupling bifurcations [11], and higher codimension phenomena [12, 13].

Since alternating period-doubling cascades are infinite cascades, we turn to renormalisation theory in order to describe their limiting behaviour. We apply two-dimensional renormalisation techniques to study in detail one type of alternating period-doubling cascade, and describe how our analysis could be adapted to study other such cascades. We find and analyse fixed points of a two-dimensional renormalisation operator, from which we derive two universal spatial scaling constants for the alternating period-doubling cascade. Using a combination of analytical and numerical approaches, we then find relevant eigenvalues and eigenfunctions of the linearisation of the renormalisation operator, from which we are able to derive universal parameter scalings for the alternating perioddoubling cascade. We show how two unstable eigenvalues of the renormalisation problem mean that an alternating period-doubling cascade will only occur at a critical value of a second parameter, and describe how the cascade unfolds for nearby values of the second parameter. We illustrate all these results with an example, from which we derive values that are close to the theoretically predicted universal constants.

In Section 2, we provide a definition of an alternating period-doubling cascade and show that such a structure cannot occur in a one-dimensional map. For two-dimensional maps, we show that alternating period-doubling cascades are can occur with only two different stability configurations. A numerical example of one of these types is also considered. The renormalisation analysis of one type of alternating period-doubling cascade is presented in Section 3, with good agreement between the theory and numerical results being obtained for the universal scaling constants. Finally, Section 4 contains conclusions and suggestions for future work. 


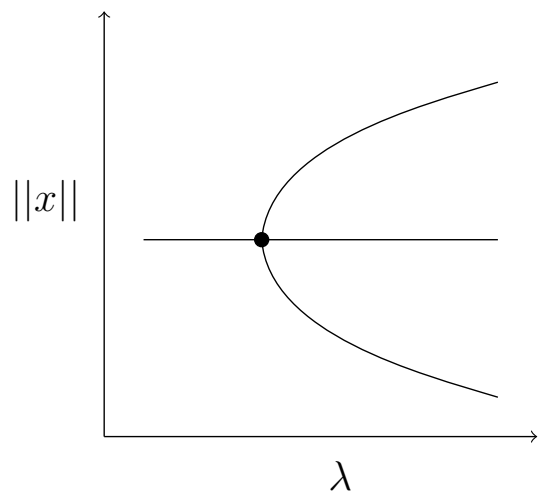

(a) A forward period-doubling bifurcation.

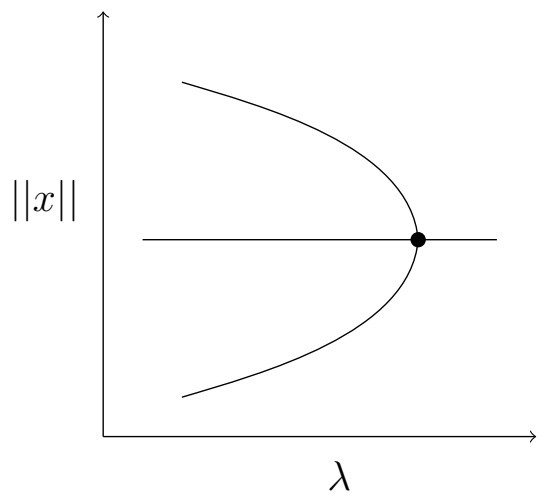

(b) A backward period-doubling bifurcation.

FiguRE 1. Forward and backward period-doubling bifurcations.

\section{Alternating Period-Doubling Cascades}

We consider a map

$$
x_{n+1}=f\left(x_{n}, \lambda\right),
$$

where $f: \mathbb{R}^{m} \times \mathbb{R} \rightarrow \mathbb{R}^{m}$ is a smooth function. We suppose that $f$ has a branch of fixed points $(x(\lambda), \lambda)$, where $x(\lambda)$ is found by solving the fixed point equation

$$
x(\lambda)=f(x(\lambda), \lambda) .
$$

We begin by recalling a familiar definition [1]:

Definition 2.1. Let $(x, \lambda)=\left(x\left(\lambda_{0}\right), \lambda_{0}\right)$ be a fixed point of $f$. The map (1) is said to undergo a period-doubling bifurcation at $\left(x\left(\lambda_{0}\right), \lambda_{0}\right)$ if the Jacobian matrix has a simple eigenvalue which crosses -1 at $(x, \lambda)=\left(x\left(\lambda_{0}\right), \lambda_{0}\right)$, and no other eigenvalues of the Jacobian matrix lie on the unit circle at this point.

Emerging from a period-doubling bifurcation along a branch of fixed points we observe two connected branches of period 2 solutions. We are interested in describing the direction in which bifurcating branches of solutions emerge from period-doubling bifurcation points, and so we provide the following definition:

Definition 2.2. Let $\left(x\left(\lambda_{0}\right), \lambda_{0}\right)$ be a period-doubling bifurcation point for the map (1). We say that the period-doubling bifurcation is a forward period-doubling bifurcation if there exists $\varepsilon>0$ such that the bifurcating period two solutions exist in a small neighbourhood of the bifurcation point for $\lambda \in\left(\lambda_{0}, \lambda_{0}+\varepsilon\right]$ but not for $\lambda \in\left[\lambda_{0}-\varepsilon, \lambda_{0}\right)$. Similarly we say that the period-doubling bifurcation is a backward period-doubling bifurcation if the period two solutions exist in a small neighbourhood of the bifurcation point for $\lambda \in\left[\lambda_{0}-\varepsilon, \lambda_{0}\right)$ but not for $\lambda \in\left(\lambda_{0}, \lambda_{0}+\varepsilon\right]$.

The bifurcation diagrams for typical forward and backward period-doubling bifurcations are shown in Fig. 1. We note that the definitions of forward and backward period-doubling bifurcations can also be extended in a natural way to definitions of forward and backward period-doubling cascades.

Although Definition 2.1 is formulated in terms of a period-doubling bifurcation along a branch of fixed points, the definition can clearly be extended to branches of solutions of arbitrary period. We also observe that Definition 2.2 is independent of the period of the branch of solutions on which the period-doubling bifurcation occurs. 
In contrast to the more familiar properties of supercriticality and subcriticality, no reference is made to stability in the definition of forward and backward period-doubling bifurcations. Indeed, forward and backward period-doubling bifurcations may be either supercritical or subcritical. Some of these properties are made more concrete in the following example.

Example 2.3. The standard logistic map is defined by

$$
x_{n+1}=f\left(x_{n}, \lambda\right)=\lambda x_{n}\left(1-x_{n}\right),
$$

where $x_{n} \in[0,1]$ for all $n$, and $\lambda \in[0,4]$ and has a branch of fixed points given by

$$
x(\lambda)=\frac{\lambda-1}{\lambda} .
$$

A supercritical period-doubling bifurcation occurs on the branch of fixed points at $(x, \lambda)=$ $\left(\frac{2}{3}, 3\right)$. According to Definition 2.2, this is also a forward period-doubling bifurcation, and is the first step in a forward period-doubling cascade.

Now consider the closely related map defined by

$$
x_{n+1}=g\left(x_{n}, \lambda\right)=(4-\lambda) x_{n}\left(1-x_{n}\right),
$$

where again $x_{n} \in[0,1]$ for all $n$, and $\lambda \in[0,4]$. The bifurcation diagram for this map is obtained by reflecting the bifurcation diagram for the logistic map about the point $\lambda=2$. Thus, there is again a branch of fixed points with a period-doubling bifurcation at $(x, \lambda)=\left(\frac{2}{3}, 1\right)$ which is again supercritical but in this case is a backward period-doubling bifurcation and is the first step in a backward period-doubling cascade.

The example above illustrates that a forward period-doubling bifurcation can be transformed to a backward period-doubling bifurcation (and vice-versa) by the simple coordinate change $\lambda \rightarrow-\lambda$. Thus, the concept of forward and backward period-doubling bifurcations is not particularly significant in the context of isolated period-doubling bifurcations - maps related by such a simple change of co-ordinates are not typically considered to be distinct objects - and so a property which is not invariant under such a transformation is not of great interest. However, we will use Definition 2.2 to compare the direction in which bifurcating branches of solutions emerge for two successive period-doubling bifurcations within a period-doubling cascade.

Definition 2.4. We say that a period-doubling cascade is an alternating period-doubling cascade if, given any two consecutive period-doubling bifurcations in the cascade, one is a forward period-doubling bifurcation and the other is a backward period-doubling bifurcation.

A section of the bifurcation diagram of a typical alternating period-doubling cascade is given in Fig. 2, where period-doubling bifurcation points are marked by heavy circles. For the sake of clarity we show only the upper branch of higher-period solution branches which emerge from each bifurcation point. We note that an alternating period-doubling cascade as a whole is invariant under the coordinate change $\lambda \rightarrow-\lambda$.

Before proceeding to analyse the properties of alternating period-doubling cascades, we note that we could also define "composite" period-doubling cascades where, for example, the first $n_{1}$ bifurcations are forward period-doubling bifurcations, the next $n_{2}$ bifurcations alternate between forward and backward period-doubling bifurcations, the next $n_{3}$ bifurcations are backward period-doubling bifurcations, and so on. While in this paper we focus mainly on alternating period-doubling cascades, we will also show how these can unfold into composite cascades which start with alternating bifurcations and then go either forward or backward. 


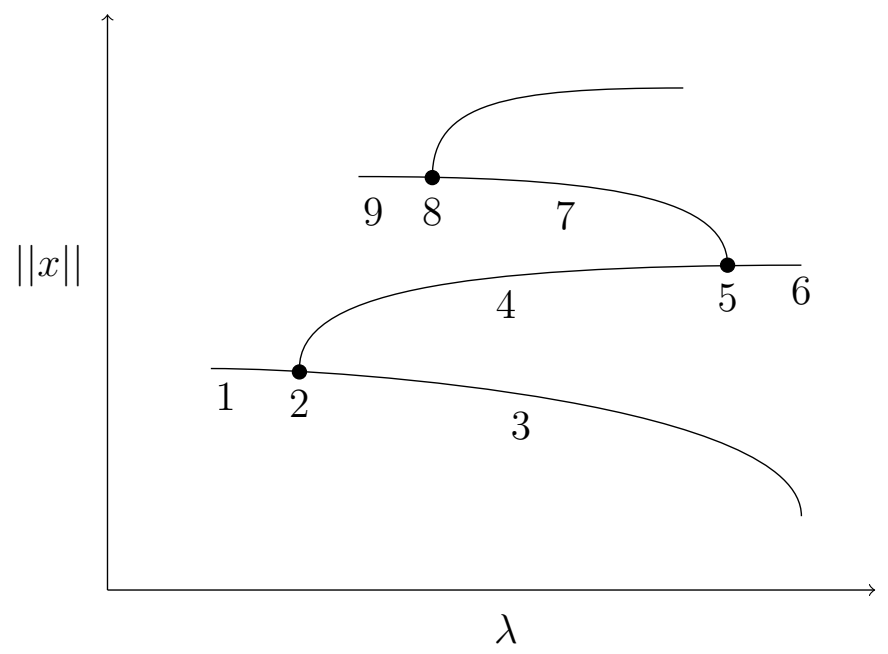

FiguRE 2. A typical section of the bifurcation diagram for an alternating period-doubling cascade. The black circles indicate period-doubling bifurcation points.

In our study of these cascades, we shall assume that no limit points occur on a branch of solutions between successive period-doubling bifurcations, so that the bifurcation diagram given in Fig. 2 is a qualitatively accurate representation of the alternating period-doubling cascades we study.

The geometric structure of an alternating period-doubling cascade restricts how the eigenvalues of the linearisation of the underlying map can change as we proceed throughout the cascade. In particular, we recall the following well known facts:

(F1) At a period-doubling bifurcation point a simple eigenvalue crosses the unit circle at -1 .

(F2) At a limit point a simple eigenvalue crosses the unit circle at +1 .

(F3) In a neighbourhood of a forward (resp. backward) period-doubling bifurcation point, the number of stable eigenvalues along the bifurcating branch is the same as the number of stable eigenvalues along the branch of lower period immediately before (resp. after) the bifurcation point.

(F4) In the limit as a period-doubling bifurcation point is approached along the higher period branch which emerges from it, the eigenvalues are the square of the eigenvalues on the branch of lower period at the same point.

We also recall our earlier assumption:

(A1) No limit points occur between two successive period-doubling bifurcations. These observations lead immediately to the following result:

Theorem 2.5. A one-dimensional map cannot generate an alternating period-doubling cascade.

Proof. For a one-dimensional map, there is a single eigenvalue $\sigma$ that determines stability of each periodic point and any bifurcations that occur. Consider a particular periodic point on a given branch of periodic solutions which lies between two period-doubling bifurcation points (such as point 4 in Fig. 2). Then there are only three possible cases for the value of the eigenvalue $\sigma$ at that point, namely $\sigma>1,-1<\sigma<1$ or $\sigma<-1$. We consider each of these cases in turn and show that the geometric structure of an alternating period-doubling cascade, as shown in Fig. 2, is not possible in each case.

If $\sigma>1$ then, since $\sigma$ cannot move away from the real axis, it must cross the unit circle at +1 before it reaches (and passes through) -1 to give the next period-doubling 
bifurcation. However, by (F2), this implies that a limit point occurs before the perioddoubling bifurcation which violates our assumption (A1).

If $-1<\sigma<1$, then the next period-doubling bifurcation must be subcritical, since the alternating structure always requires the bifurcating branch to turn back over the earlier part of the cascade, and so the bifurcating branch is initially unstable. Since the eigenvalue on the bifurcating branch at the bifurcation point is $\sigma=1$ by (F4), then we must have $\sigma>1$ initially along the bifurcating branch. As in the previous case, this eigenvalue must pass through the unit circle at +1 before it reaches -1 for another period-doubling bifurcation, again contradicting (A1).

Finally, when $\sigma<-1$, our point of interest is unstable, and so the next period-doubling bifurcation must be supercritical, again due to the alternating structure. Thus, the bifurcating branch is initially stable and so, on the bifurcating branch, we have $-1<\sigma<1$. This is the same situation as considered previously except that it is one branch further up the cascade.

Since alternating period-doubling cascades cannot occur in one-dimensional maps we turn our attention to the next simplest case, namely that of two-dimensional maps. In this setting there are two eigenvalues along each branch of solutions. This allows more interesting behaviour to occur, including eigenvalues appearing as a complex conjugate pair.

Our next aim is to describe the typical behaviour of two-dimensional alternating perioddoubling cascades by tracking the locations of the eigenvalues as we pass through the cascade. We will be able to do this using the restrictions (F1)-(F4), the assumption (A1) and our assumption that Fig. 2 provides a qualitatively accurate representation of the bifurcation diagram of a typical section of an alternating period-doubling cascade.

We make the preliminary observation that the location of the eigenvalues along the branch of fixed point solutions may be atypical in the context of the full infinite cascade. Along branches of solutions of period 2 and greater, there is a section of the solution branch which connects two consecutive period-doubling bifurcations. In a neighbourhood of the lower-period period-doubling bifurcation along this branch of solutions, both of the eigenvalues must lie in the right half-plane by (F4). Along the fixed point solution branch, however, this restriction clearly does not apply, and the eigenvalues may assume positions which would not be possible along any other branch of solutions in the cascade. As we are interested in the typical behaviour of alternating period-doubling cascades, we will refer to a "typical" section of an alternating period-doubling cascade to mean a section of the cascade which excludes the fixed point branch of solutions (or, alternatively, any section of a cascade where the location of the eigenvalues along the branch of fixed points is not prohibited from occurring along a branch of greater period by (F1)-(F4) and (A1)).

Theorem 2.6. There are (up to reflection and translation by period) precisely two different ways to assign eigenvalue locations through an alternating period-doubling cascade bifurcation diagram as shown in Fig. 2 which are consistent with the restrictions (F1)(F4) and the assumption (A1). These two cases are shown in Fig. 3 which we refer to as type I (Fig. 3(a)) and type II (Fig. 3(b)).

Proof. This result is proved using a similar approach to the proof of Theorem 2.5. We consider all possible qualitatively different configurations of two eigenvalues at point 1 of Fig. 2. Using the rules given in (F1)-(F4) and the geometric structure of the alternating cascade places many restrictions on the eigenvalues throughout the cascade and so all possible positions of the eigenvalues relative to the unit circle at each point along each of the branches can be determined. If the eigenvalue configuration at points 9,8 and 7 are 


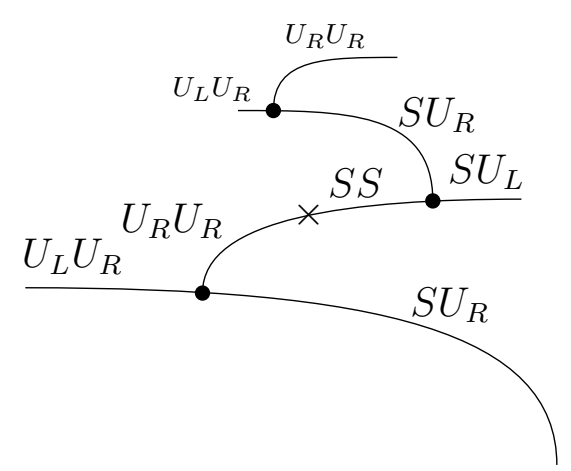

(a) A section of a type I alternating period-doubling cascade.

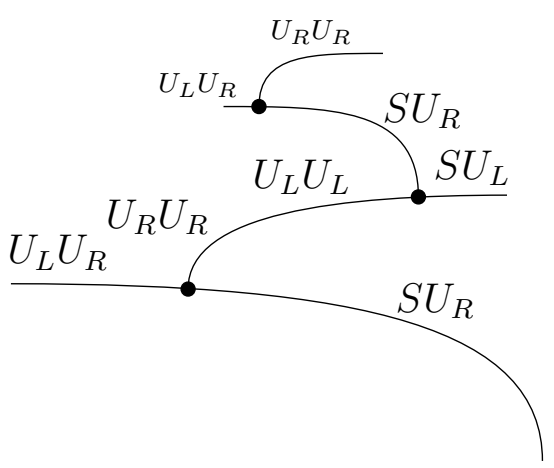

(b) A section of a type II alternating period-doubling cascade.

FiguRE 3. Two possible alternating period-doubling cascades. The black circles indicate period-doubling bifurcation points and the crosses indicate Neimark-Sacker bifurcations.

the same as at points 1, 2 and 3 respectively, then we accept this as a valid alternating period-doubling cascade. Using this approach, we find that there are only two eigenvalue configurations that meet this criterion which are shown in Fig. 3, where $S$ represents a stable eigenvalue inside the unit circle, $U_{R} / U_{L}$ represents an unstable eigenvalue to the right/left of the unit circle and a cross indicates a Neimark-Sacker bifurcation (where a complex conjugate pair of eigenvalues cross the unit circle). The movement of the eigenvalues through each of these cascades is shown in Fig. 4.

The qualification "up to reflection and translation by period" in the statement of Theorem 2.6 refers to the fact that each of the assignments of eigenvalue locations given on the bifurcation diagrams in Fig. 3 can be "translated" through their respective perioddoubling cascades by one period-doubling bifurcation (so that each label, which refers to a point on a branch of solutions of period $2^{k}$ for some value of $k$, is shifted to the branch of solutions of period $2^{k+1}$ ). The result will be another bifurcation diagram which provides a valid assignment of eigenvalue locations to Fig. 2. Similarly, each bifurcation diagram given in Fig. 3 can be reflected horizontally to obtain another valid bifurcation diagram. We consider these bifurcation diagrams to represent variations of a single alternating period-doubling cascade rather than distinct alternating period-doubling cascades.

We note that the requirement for a valid alternating period-doubling cascade is that the eigenvalues at points 9 and 7 of Fig. 2 are in qualitatively the same positions (relative to the unit circle) as the eigenvalues at points 1 and 3 respectively, and so an infinite alternating period-doubling cascade can be generated from the small section shown in either of the bifurcation diagrams in Fig. 3 by repeating the same eigenvalue movements at ever increasing periods. Since these two diagrams represent (up to reflection and translation by period) the only valid assignments of eigenvalue locations to a typical section of an alternating period-doubling cascade bifurcation diagram, all valid full twodimensional alternating period-doubling cascades must be generated by concatenating copies of one of the two bifurcation diagrams given in Fig. 3.

We now consider conditions for the existence of alternating period-doubling cascades in area/volume-preserving maps and systems of differential equations. 


\section{Theorem 2.7.}

(i) A two-dimensional area-preserving map cannot generate an alternating perioddoubling cascade, but a three-dimensional volume-preserving map can generate such a cascade.

(ii) A system of four or more first order autonomous differential equations or a system of three or more first order periodically-forced differential equations can give rise to an alternating period-doubling cascade. However, systems of differential equations of lower order cannot give rise to an alternating period-doubling cascade.

Proof. It is well known that a two-dimensional area-preserving map can have a perioddoubling cascade $[9,10]$. However, we now show that it cannot have an alternating period-doubling cascade.

For an area-preserving map, the determinant of the Jacobian evaluated at a fixed point is 1 , with obvious generalisations to periodic points. Thus, for a two-dimensional map, the product of the two eigenvalues must always be 1 . This implies in particular that the map cannot have an eigenvalue of zero.

A system of $n+1$ first order autonomous differential equations gives rise to an $n$ dimensional Poincaré map. Similarly, a system of $n$ first order periodically-forced differential equations also gives rise to an $n$-dimensional stroboscopic map. In both cases the map is at least locally a diffeomorphism and hence is invertible. This again excludes the possibility of a zero eigenvalue of the Jacobian evaluated at a periodic point.

If $n=1$, then either system of differential equations will give rise to a one-dimensional map, and by Theorem 2.5, this means that an alternating period-doubling cascade is not possible.

If $n=2$ or for an area-preserving map, then we have a two-dimensional map, but where a zero eigenvalue is not permitted. Now from Fig. 4(a),(b), it can be seen that on the period 2 branch for both types of alternating period-doubling cascade, a real eigenvalue must pass through zero. Thus, an alternating period-doubling cascade is not possible in these cases either.

If $n=3$ or for a three-dimensional volume-preserving map, we have a three-dimensional map. Suppose that the third eigenvalue on the period 2 branch is real and lies between 0 and 1 . In this case, it is possible to have a collision of two real eigenvalues which become complex, move around the origin, and collide again on the real axis between -1 and 0 , at which point one of them can proceed to cross the circle at -1 giving the required period-doubling bifurcation point. Thus, the problem of a zero eigenvalue can be avoided in these cases. In the case of the volume-preserving map, there is also a requirement that the modulus of the product of the eigenvalues is one, which means that there cannot be three stable or three unstable eigenvalues. It can be shown that adding a third eigenvalue $\sigma_{3}$ satisfying $\sigma_{3}>1$ for a type I cascade or $0<\sigma_{3}<1$ for a type II cascade on the lowest period branch in Fig. 3, all the required conditions can be satisfied to retain these two types of cascade. However, the type I cascade will no longer involve a Neimark-Sacker bifurcation. Thus, we conclude that alternating period-doubling cascades can occur in these cases.

A key difference between the type I and type II alternating period-doubling cascades is the presence (or absence) of Neimark-Sacker bifurcations, and consequently the presence (or absence) of sections of solution branches which are stable. In particular, the type II alternating period-doubling cascade has no stable solutions, whereas every second branch of solutions in the type I alternating period-doubling cascade has a section (between a Neimark-Sacker bifurcation and a period-doubling bifurcation) which is stable. This implies that for the full alternating period-doubling cascade, there is a nested sequence 
Period 1

branch:

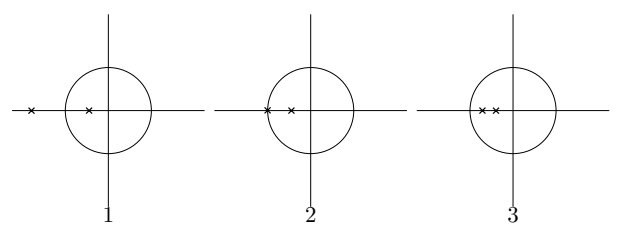

Period 2

branch:
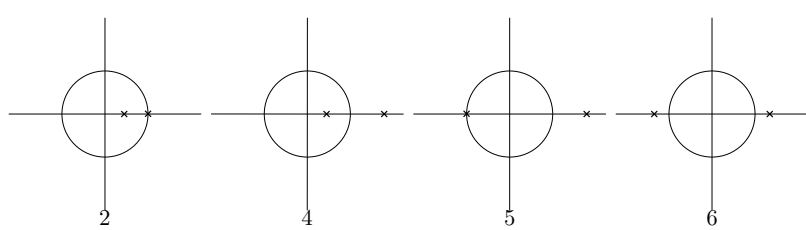

Period 4

branch:

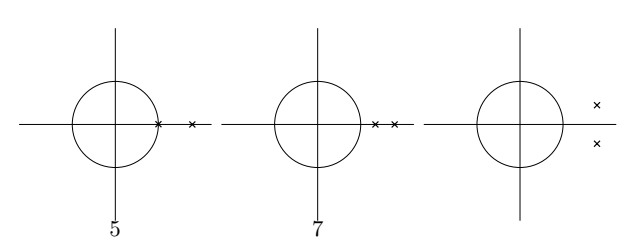

(a) The eigenvalue movements for the type I alternating period-doubling cascade up to the point where the eigenvalues become complex.

Period 1

branch:

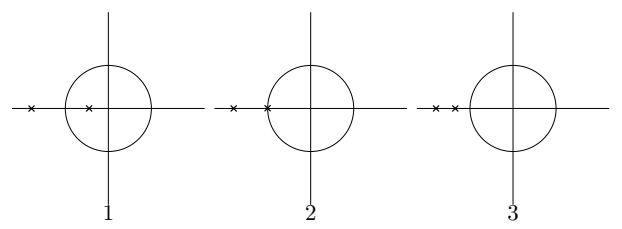

Period 2

branch:

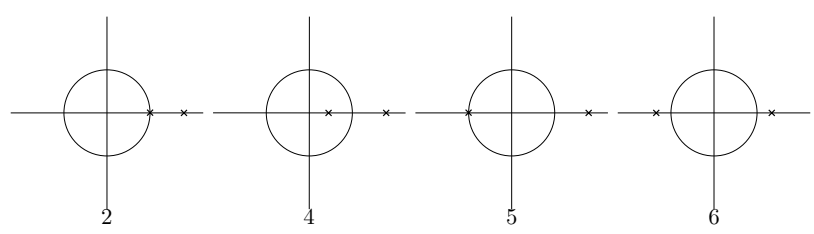

Period 4

branch:

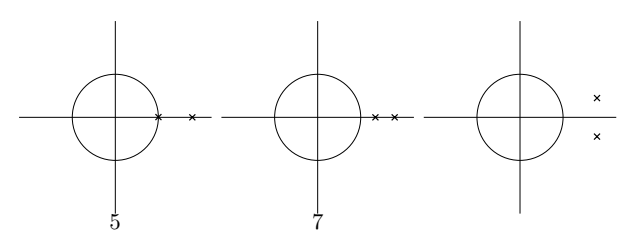

(b) The eigenvalue movements for the type II alternating period-doubling cascade up to the point where the eigenvalues become complex.

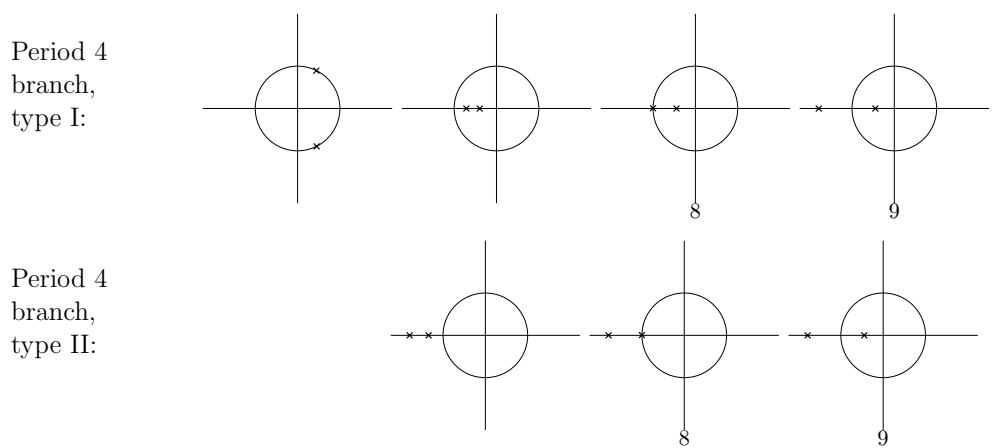

(c) The remaining eigenvalue movements for the type I and type II cascades.

FiguRE 4. Eigenvalue movements through the type I and type II alternating period-doubling cascades. 


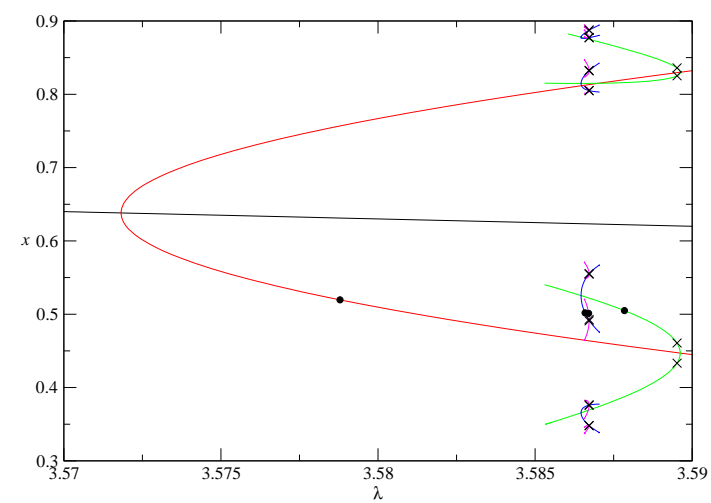

(a) The $x-\lambda$ projection.

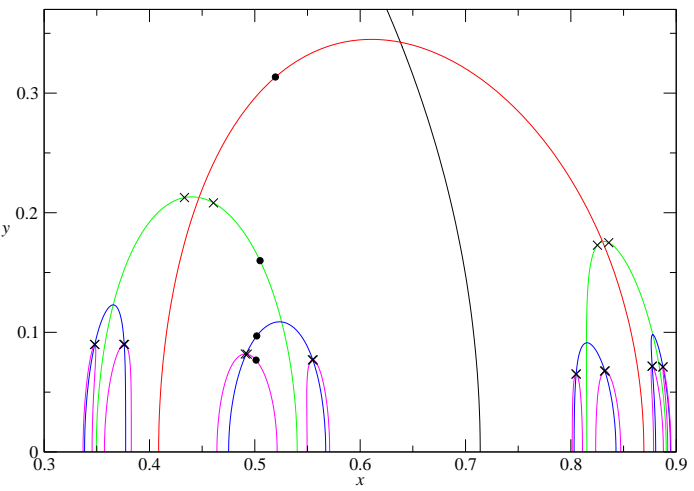

(c) The $x-y$ projection.

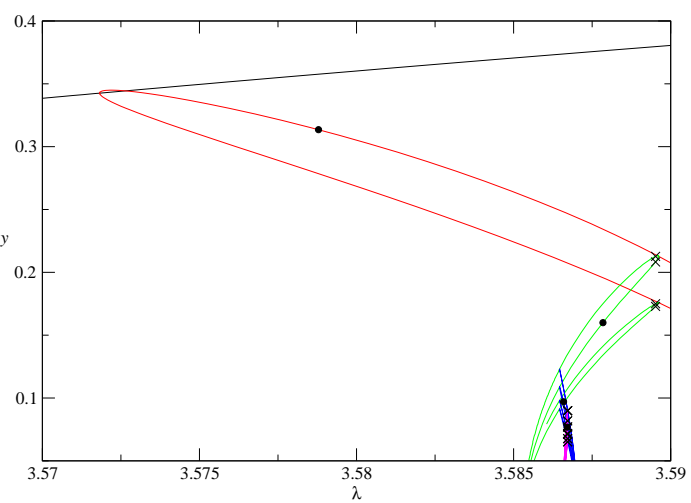

(b) The $y-\lambda$ projection.

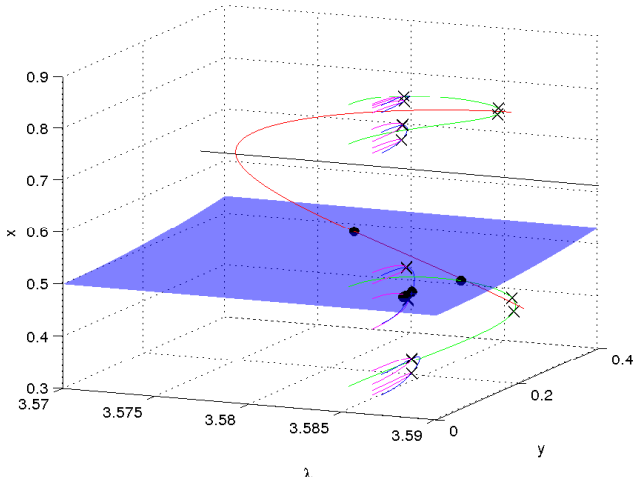

(d) A three-dimensional view.

FiguRE 5. The low-period solution branches of equations (2). Black: period 1; red: period 2; green: period 4; blue: period 8; magenta: period 16 . The crosses indicate Neimark-Sacker bifurcation points. The black circles show points at which there is a zero eigenvalue. The surface shown in $(d)$ is the plane on which the Jacobian matrix is singular.

of parameter intervals in which there are stable periodic solutions, and hence there are many co-existing stable periodic solutions for a range of parameter values.

Example 2.8. We consider the system of equations

$$
\begin{aligned}
& x_{n+1}=F_{1}\left(x_{n}, y_{n}, \lambda\right)=\lambda x_{n}\left(1-x_{n}\right)-y_{n}^{2}\left(0.5050541762+1.7 x_{n}\right), \\
& y_{n+1}=F_{2}\left(x_{n}, y_{n}, \lambda\right)=y_{n}\left(\lambda-3.21+x_{n}\right) .
\end{aligned}
$$

This system of equations exhibits an alternating period-doubling cascade as we vary $\lambda$. The low-period branches (up to period 16) are shown in Fig. 5 while some of the higherperiod branches (up to period 64) are shown in Fig. 6. The bifurcation points in the cascade up to the branch of period 1024 are shown in Table 1. These were found approximately using AUTO [14] and Maple was then used to find the bifurcation points to high accuracy. It can be seen that the difference between the bifurcation points does indeed alternate in sign and the magnitude of the difference is decreasing. Thus, there will be a limit of the sequence of bifurcation points $\lambda_{\infty}$ which can be approximated by assuming that

$$
\lambda_{\infty}-\lambda_{n}=c\left(\lambda_{\infty}-\lambda_{n-1}\right)
$$




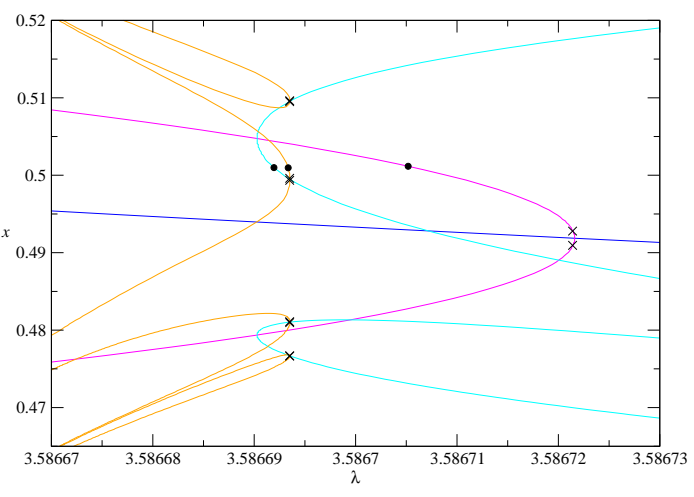

(a) The $x-\lambda$ projection.

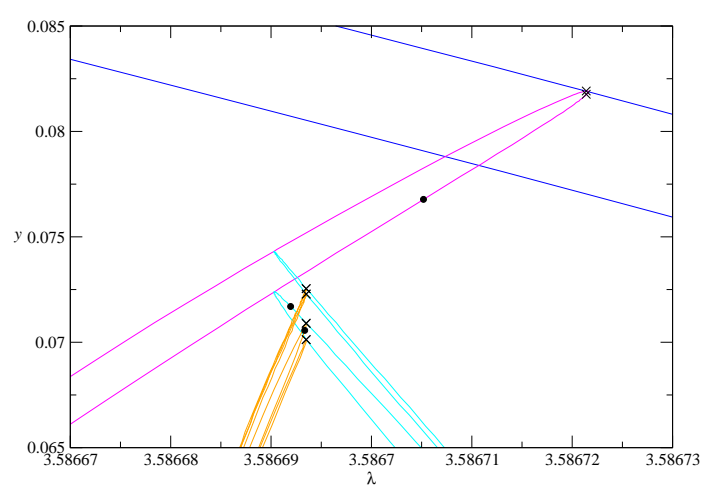

(b) The $y-\lambda$ projection.

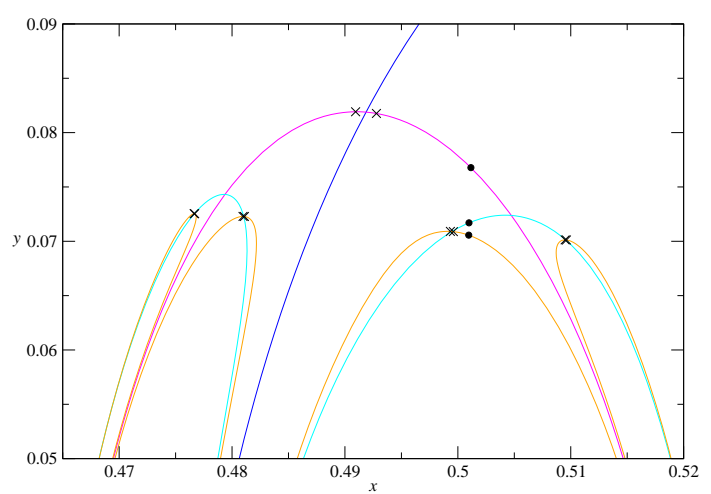

(c) The $x-y$ projection.

Figure 6. Higher-period solution branches of equations (2). Blue: period 8; magenta: period 16; cyan: period 32; orange: period 64 . The crosses indicate Neimark-Sacker bifurcation points. The black circles show points at which there is a zero eigenvalue.

for some (negative) constant $c$. Using this assumption and eliminating $c$ gives

$$
\lambda_{\infty}=\frac{\lambda_{n-1}^{2}-\lambda_{n} \lambda_{n-2}}{2 \lambda_{n-1}-\left(\lambda_{n}+\lambda_{n-2}\right)} .
$$

Taking $n=10$ gives the approximation to $\lambda_{\infty}$ shown in Table 1 .

For this example, there are stable periodic points on branches of period 1, 4, 16, etc. and no stable solutions on the other branches. However, the length of the parameter interval on which there are stable periodic points shrinks rapidly as the period increases. The length of the stable intervals on the branches of period 4, 16 and 64 is $3.06 \times 10^{-3}$, $3.12 \times 10^{-5}$ and $3.99 \times 10^{-7}$ respectively.

If we choose a parameter value at which there are stable periodic solutions on two or more branches through the cascade, then we note from Fig. 3(a) that the alternate branches have stability $S U_{R}$, and hence are saddles. In the neighbourhood of a perioddoubling bifurcation from an $S S$ branch to an $S U_{R}$ branch, there will be a heteroclinic connection between the bifurcating branch and the lower period stable branch, as a consequence of the centre manifold reduction that can be done at the bifurcation point, and this corresponds to the unstable manifold of the saddle point. This manifold is likely to persist further from the bifurcation point, provided that the Neimark-Sacker bifurcation on the $S S$ branch and any bifurcating invariant circles are not reached. The stable manifold of the branch of saddle points will then act as the boundary between the basins of 


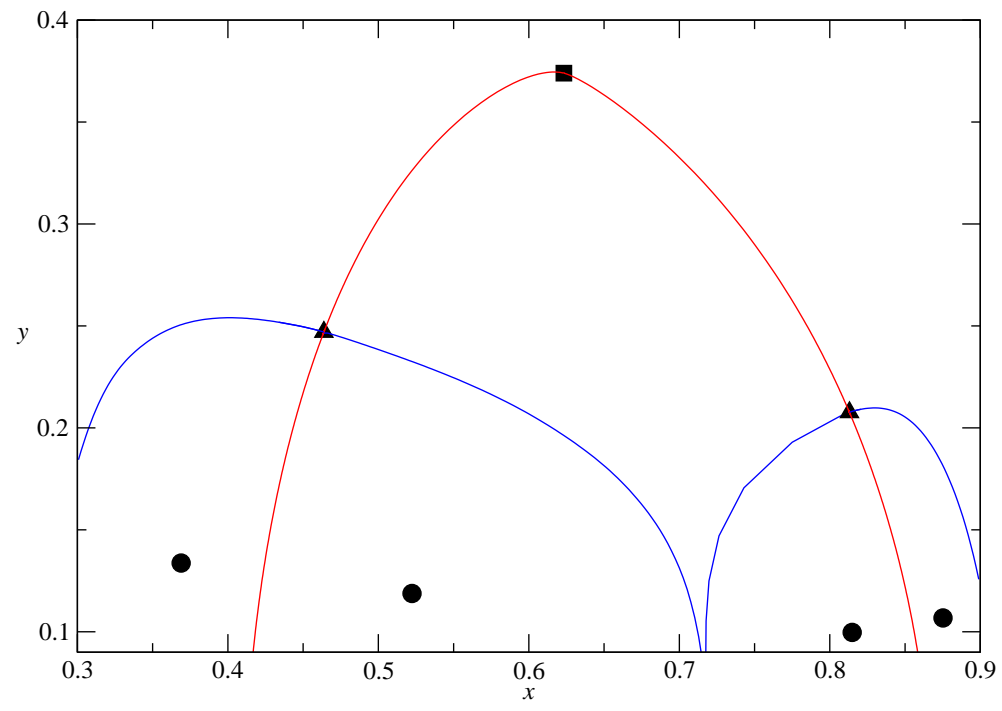

Figure 7. Section of the phase portrait for (2), showing the fixed point (square), period 2 solutions (triangles) and period 4 solutions (circles). The stable (blue) and unstable (red) manifolds of the period 2 saddles are also shown.

attraction of the two stable periodic points. This situation is illustated in Fig. 7 for stable period 1 and 4 points and saddle period 2 points.

It is apparent in our example that the coefficient of $y_{n}^{2}$ in the first equation of (2) has been very carefully chosen, and it is natural to ask what happens if this value is changed. If this value is increased slightly then we observe a period-doubling cascade which alternates at low period, but after some point it exhibits only forward period-doubling bifurcations. Conversely, if this coefficient is decreased slightly, then again there is an alternating period-doubling cascade at low period but with a backward period-doubling cascade after some point. Indeed, if the value of this coefficient is increased further (to a value of 2.5, for example) then the system of equations (2) exhibits a forward period-doubling cascade, whereas if the coefficient is decreased sufficiently (to -0.7 , for example) then we observe a backward period-doubling cascade. Thus, the alternating period-doubling cascade is the midpoint in a transition from a forward to a backward period-doubling cascade and, as such, will only occur at isolated values of a second parameter. A more detailed analysis of a two-parameter unfolding of the alternating period-doubling cascade is considered elsewhere $[15,16]$.

\section{RenORMALisAtion}

3.1. Introduction. Feigenbaum $[3,4,5]$ analysed the limiting behaviour of period-doubling cascades in one-dimensional maps using renormalisation theory, which we briefly review before applying this method to the analysis of the limiting behaviour of an alternating period-doubling cascade.

Consider the one-dimensional iteration

$$
x_{n+1}=f\left(x_{n}, \lambda\right), \quad f: \mathbb{R} \times \mathbb{R} \rightarrow \mathbb{R}
$$

and assume that $f$ has a single quadratic maximum at $x=0$ for all $\lambda$. Renormalisation theory focusses on the superattractive points on each successive periodic branch and these occur when $x=0$ is one of the periodic points on the cycle, since the derivative of $f$ is zero at this point, and so are defined by

$$
f^{2^{r}}\left(0, \lambda_{r}\right)=0, \quad r=0,1,2, \ldots
$$




\begin{tabular}{|c|c|c|r|}
\hline$n$ & Period & $\lambda_{n}$ & \multicolumn{1}{|c|}{$\lambda_{n}-\lambda_{n-1}$} \\
\hline 0 & 1 & 3.57182321887724 & - \\
1 & 2 & 3.58961762945040 & $1.7794 \times 10^{-2}$ \\
2 & 4 & 3.58646379318567 & $-3.1538 \times 10^{-3}$ \\
3 & 8 & 3.58672156873512 & $2.5778 \times 10^{-4}$ \\
4 & 16 & 3.58669024548849 & $-3.1323 \times 10^{-5}$ \\
5 & 32 & 3.58669350041468 & $3.2549 \times 10^{-6}$ \\
6 & 64 & 3.58669313853877 & $-3.6188 \times 10^{-7}$ \\
7 & 128 & 3.58669317764867 & $3.9110 \times 10^{-8}$ \\
8 & 256 & 3.58669317336413 & $-4.2845 \times 10^{-9}$ \\
9 & 512 & 3.58669317382887 & $4.6474 \times 10^{-10}$ \\
10 & 1024 & 3.58669317377776 & $-5.1106 \times 10^{-11}$ \\
$\vdots$ & $\vdots$ & $\vdots$ & \\
$\infty$ & $\infty$ & 3.58669317378283 & \\
\hline
\end{tabular}

TABLE 1. Bifurcation points on branches of period $2^{n}$.

The periodic point on the corresponding connected branch of solutions to this point is found by iterating $2^{r-1}$ times and so is given by $f^{2^{r-1}}\left(0, \lambda_{r}\right)$. The distance $d_{r}$ between these two periodic points is therefore

$$
d_{r}=f^{2^{r-1}}\left(0, \lambda_{r}\right)
$$

Feigenbaum observed that

$$
\lim _{r \rightarrow \infty} \frac{d_{r}}{d_{r+1}}=-\alpha
$$

where $\alpha \approx 2.503$ is Feigenbaum's spatial scaling constant.

Feigenbaum also observed that the rescaled functions

$$
(-\alpha)^{n} f^{2^{n}}\left(\frac{x}{(-\alpha)^{n}}, \lambda_{n+r}\right)
$$

converge as $n \rightarrow \infty$. If we define

$$
g_{r}(x)=\lim _{n \rightarrow \infty}(-\alpha)^{n} f^{2^{n}}\left(\frac{x}{(-\alpha)^{n}}, \lambda_{n+r}\right)
$$

then it can be shown that

$$
g_{r-1}=T g_{r}
$$

where the nonlinear scaling operator $T$ is defined by

$$
T \phi(x)=-\alpha \phi\left(\phi\left(-\frac{x}{\alpha}\right)\right) .
$$

The functions $g_{r}(x)$ also converge as $r \rightarrow \infty$ and we define

$$
g(x)=\lim _{r \rightarrow \infty} g_{r}(x) .
$$

The function $g$ corresponds to a fixed point of the operator $T$ and hence satisfies

$$
g=T g \text {. }
$$

The solutions of (5) are not unique since if $g(x)$ is a solution, then so is $\gamma g(x / \gamma)$ for all $\gamma \neq 0$. A unique solution of this equation is found by adding the extra equation

$$
g(0)=1 \text {. }
$$


The equations (5) and (6) can then be solved for $g(x)$ and $\alpha$. The solution of these equations can only be found numerically, and is given by $\alpha=2.50290788 \ldots$ and $g(x)=$ $f_{F}(x)$, where the Feigenbaum function $f_{F}(x)$ is given approximately by [4]

$$
f_{F}(x)=1-1.52763300 x^{2}+0.10481519 x^{4}+0.02670567 x^{6}+O\left(x^{8}\right)
$$

The Fréchet derivative $J_{g}$ of the operator $T$ is given by

$$
J_{g} \psi=-\alpha\left[g^{\prime}\left(g\left(-\frac{x}{\alpha}\right)\right) \psi\left(-\frac{x}{\alpha}\right)+\psi\left(g\left(-\frac{x}{\alpha}\right)\right)\right] .
$$

The largest eigenvalue in magnitude of the linear operator $J_{f_{F}}$ is found numerically to be $[17]$

$$
\delta=4.66920161 \ldots
$$

We computed the corresponding eigenfunction to be

$$
\psi_{\delta}=1-0.32564092 x^{2}-0.05055612 x^{4}+0.01456495 x^{6}+O\left(x^{8}\right)
$$

which is similar to that given in [4]. We note that $-\alpha$ is also an eigenvalue of $J_{f_{F}}$ with eigenfunction $\psi_{\alpha}(x)=f_{F}^{\prime}(x)-1$, which arises from the coordinate change $x \rightarrow x+\epsilon$. Feigenbaum also showed that the limiting parameter scaling is given by

$$
\lim _{r \rightarrow \infty} \frac{\lambda_{r+1}-\lambda_{r}}{\lambda_{r+2}-\lambda_{r+1}}=\delta
$$

The spatial scaling constant $\alpha$ and the parameter scaling constant $\delta$ are found to be the same for a wide class of maps, and so are referred to as universal constants.

Feigenbaum's work on one-dimensional renormalisation problems has been extended to higher dimensions in a variety of ways $[9,12,13,18]$. In [12], the authors begin by discussing systems with unidirectional coupling, and later expand their scope to consider more general two-dimensional renormalisation phenomena using a two-dimensional renormalisation operator. Two dimensional renormalisation is also considered in [13], where the authors provide a survey of many of the extensions to the renormalisation theory since Feigenbaum's work. More exotic constructions, such as Hénon-like maps [19] and maps with "pacemakers" [20], have also been discussed in the literature.

The renormalisation operator for two-dimensional problems is given by

$$
S\left(\begin{array}{c}
\phi(x, y) \\
\psi(x, y)
\end{array}\right)=\left(\begin{array}{c}
\gamma \phi^{2}\left(\frac{x}{\gamma}, \frac{y}{\eta}\right) \\
\eta \psi^{2}\left(\frac{x}{\gamma}, \frac{y}{\eta}\right)
\end{array}\right),
$$

A fixed point of the operator $S$ comprises a pair of functions $f, g: \mathbb{R}^{2} \rightarrow \mathbb{R}$ such that

$$
\left(\begin{array}{l}
f \\
g
\end{array}\right)=S\left(\begin{array}{l}
f \\
g
\end{array}\right)
$$

As in the one-dimensional case, solutions of this equation are not unique. In particular, if $(f, g)$ is a fixed point of the operator $S$ then the functions $\left(\kappa f\left(\frac{x}{\kappa}, \frac{y}{\theta}\right), \theta g\left(\frac{x}{\kappa}, \frac{y}{\theta}\right)\right)$ are also fixed points for all $\kappa, \theta \neq 0$. To obtain a unique solution, now requires the two normalisation conditions

$$
f(0,0)=g(0,0)=1 .
$$

Equations (8) and (9) can then be solved for $f, g, \gamma, \eta$.

Numerically, we solved the one-dimensional and two-dimensional renormalisation problems with a pseudo-spectral collocation method using Chebyshev polynomials [21, 22]. The code was written in Maple so that the numerical accuracy could be increased. For one-dimensional problems, we used a basis consisting of 21 Chebyshev polynomials while for the two-dimensional problems, we used a basis of 13 Chebyshev polynomials in each space dimension, giving a total of 169 polynomials in the basis. 
3.2. Renormalisation for alternating period-doubling cascades. The alternating period-doubling cascades for two-dimensional maps described in the previous section are infinite cascades, and so we use renormalisation theory to analyse the limiting behaviour of such cascades.

Suppose that we have a map

$$
X_{n+1}=F\left(X_{n}, \lambda\right), \quad F: \mathbb{R}^{2} \times \mathbb{R} \rightarrow \mathbb{R}^{2},
$$

which exhibits an alternating period-doubling cascade. As we have a two-dimensional map, we will be looking for a solution of the two-dimensional fixed point equation (8) and we will then show how this solution relates to properties of the alternating period-doubling cascade.

We start by rewriting (8) as

$$
G(X)=\Gamma G\left(G\left(\Gamma^{-1} X\right)\right)
$$

where

$$
G=\left(\begin{array}{l}
f \\
g
\end{array}\right), \quad X=\left(\begin{array}{l}
x \\
y
\end{array}\right), \quad \Gamma=\left(\begin{array}{ll}
\gamma & 0 \\
0 & \eta
\end{array}\right)
$$

We first consider properties that solutions of this equation must satisfy.

Lemma 3.1. If $G(X)$ is a solution of the fixed point equation (10) then either $\operatorname{det}\left(G_{X}(0,0)\right)=$ 0 or $\operatorname{det}\left(G_{X}(G(0,0))\right)=1$.

Proof. Differentiating equation (10) and evaluating at $(x, y)=(0,0)$ gives

$$
G_{X}(0,0)=\Gamma G_{X}(G(0,0)) G_{X}(0,0) \Gamma^{-1} .
$$

Taking the determinant of each side we obtain

$$
\begin{aligned}
\operatorname{det}\left(G_{X}(0,0)\right) & =\operatorname{det} \Gamma \operatorname{det}\left(G_{X}(G(0,0))\right) \operatorname{det}\left(G_{X}(0,0)\right) \operatorname{det} \Gamma^{-1} \\
& =\operatorname{det}\left(G_{X}(G(0,0))\right) \operatorname{det}\left(G_{X}(0,0)\right)
\end{aligned}
$$

since $\operatorname{det} \Gamma^{-1}=(\operatorname{det} \Gamma)^{-1}$, whence the result follows.

To be consistent with the standard Feigenbaum theory, we will consider solutions of the fixed point equation (10) that satisfy the first condition of Lemma 3.1, namely $\operatorname{det}\left(G_{X}(0,0)\right)=0$. This implies that we should look for a sequence of points through the alternating period-doubling cascade at which the Jacobian matrix evaluated at a periodic point is singular, which in turn implies that there should be a zero eigenvalue.

For our two types of alternating period-doubling cascades, the eigenvalue movements through the cascades are shown in Fig. 4. We note that on what is labelled as the "Period 2 branch", there is an eigenvalue that starts at +1 as the branch bifurcates from a period-doubling point, and moves along the real axis to -1 at which point the next period-doubling bifurcation occurs. Since the other eigenvalue is outside the unit circle, this eigenvalue must pass through zero, and so there will be a point on this branch at which the determinant is singular.

On the "Period 4 branch", the eigenvalues both move outside of the unit circle on the positive real axis, collide and become a complex conjugate pair. For the type II cascade, this pair move round the outside of the unit circle and collide again on the negative real axis, with one of them then moving through -1 to give the next period-doubling bifurcation. Thus, there is never a zero eigenvalue along this branch. For the type I cascade, the complex conjugate pair of eigenvalues cross the unit circle and collide on the real axis inside the unit circle. If this collision occurs on the positive real axis, then one eigenvalue must pass through zero before it gets to -1 for the next bifurcation (assuming that the eigenvalues do not become complex again), and so there will be a point on the branch where the determinant is zero. On the other hand, if the eigenvalues collide on 
the negative real axis, there is no need for an eigenvalue to pass through zero before the next bifurcation, although this is possible.

In the three-dimensional $(x, y, \lambda)$ space, the condition $\operatorname{det} F_{X}(X, \lambda)=0$ defines a twodimensional surface and the discussion above shows that at least every other branch must cross this surface, and in some cases every branch will cross it.

In this paper, we analyse in detail only the type I alternating period-doubling cascade, and we will assume that the eigenvalues collide on the positive real axis so that there is a zero eigenvalue on every branch. In the case of a type I cascade with no zero eigenvalue on the "Period 4 branch", or for a type II cascade, the renormalisation process would have to be a mapping from one branch to another branch which occurs after two bifurcations rather than just one. However, we will not consider this possibility further.

Example 3.2. We consider again the map we studied in Example 2.8 given by (2). For this map, the complex eigenvalues collide on the real axis inside the unit circle quite close to +1 and so there are points with a zero eigenvalue on every branch. These points are shown in Table 2. The estimate of each of the limiting points as $n \rightarrow \infty$ can be obtained in the same way as $\lambda_{\infty}$ was calculated previously, using (3).

Since the singular points alternate in $\lambda$, we would expect the parameter scaling constant to be negative in this case. To estimate this constant, we define

$$
\kappa_{n}=\frac{\lambda_{n-1}-\lambda_{n-2}}{\lambda_{n}-\lambda_{n-1}}
$$

and the scaling constant is then

$$
\kappa=\lim _{n \rightarrow \infty} \kappa_{n} .
$$

The values of $\kappa_{n}$ are shown in Table 3 . We see from these results that $\kappa$ is indeed negative, and has an estimated value of $\kappa \approx-9.1$.

To determine the two spatial scaling constants, we note that in renormalisation theory, the scaling matrix is always assumed to be diagonal. For a practical example, we must work with the problem in eigenvector coordinates in order to get a diagonal scaling matrix, as the two scaling constants then denote the scaling in each eigenvector direction. In Table 2 , in addition to the periodic points $\left(x_{n}, y_{n}\right)$ at which there is a zero derivative, we also list the corresponding points $\left(x_{n}^{*}, y_{n}^{*}\right)$ which are half way around the orbit, since the branches through these two points connect at the period-doubling bifurcation point. We can then derive the spatial scalings in the $x$ and $y$ directions by finding $\delta x_{n}=x_{n}-x_{n}^{*}$ and $\delta y_{n}=y_{n}-y_{n}^{*}$. By changing to an eigenvector basis, using the Jacobian matrix of the periodic point for $n=10$, we can transform $\delta x_{n}$ and $\delta y_{n}$ into the new coordinate system, and we denote the new distances by $\delta X_{n}$ and $\delta Y_{n}$ respectively. The spatial scaling factors can then be estimated as $\gamma=\lim _{n \rightarrow \infty} \gamma_{n}$ and $\eta=\lim _{n \rightarrow \infty} \eta_{n}$ where

$$
\gamma_{n}=\frac{\delta X_{n-1}}{\delta X_{n}}, \quad \eta_{n}=\frac{\delta Y_{n-1}}{\delta Y_{n}}
$$

These values are also shown in Table 3 from which it can be seen that we have the approximate values $\gamma \approx-2.49$ and $\eta \approx-4.68$. The value of $\gamma$ is quite close to the scaling constant for one-dimensional maps, which is $-\alpha$ where $\alpha=2.50290788 \ldots$

3.3. Fixed Points of the Renormalisation Operator. We now seek to explain the above results in terms of a fixed point of the two-dimensional renormalisation operator. For the above example, we found that the rescaled form of the function $F_{1}$ evaluated at successive singular points seemed to be converging to a function of $x$ only, and with a maximum point occurring at the singular point. This suggests that the fixed point function $f$ should be a function only of $x$ with its derivative equal to zero. If this is the 


\begin{tabular}{|c|c|c|c|c|c|c|}
\hline$n$ & Period & $x_{n}$ & $x_{n}^{*}$ & $y_{n}$ & $y_{n}^{*}$ & $\lambda_{n}$ \\
\hline 1 & 2 & 0.5195754643 & 0.7568749655 & 0.3135028433 & 0.2785048063 & 3.578789066688 \\
2 & 4 & 0.5049499442 & 0.3875111797 & 0.1599120396 & 0.1750278239 & 3.587841000813 \\
3 & 8 & 0.5018258603 & 0.5470358717 & 0.0969896989 & 0.0928101822 & 3.586591269980 \\
4 & 16 & 0.5011440123 & 0.4827872515 & 0.0767916450 & 0.0782723967 & 3.586705196127 \\
5 & 32 & 0.5009974410 & 0.5082477475 & 0.0717037990 & 0.0711396685 & 3.586691898181 \\
6 & 64 & 0.5009659408 & 0.4980380409 & 0.0705628952 & 0.0707927444 & 3.586693313120 \\
7 & 128 & 0.5009592328 & 0.5021165518 & 0.0703175016 & 0.0702260679 & 3.586693158474 \\
8 & 256 & 0.5009577851 & 0.5004903681 & 0.0702644313 & 0.0703015854 & 3.586693175434 \\
9 & 512 & 0.5009574777 & 0.5011421757 & 0.0702531586 & 0.0702384347 & 3.586693173601 \\
10 & 1024 & 0.5009574113 & 0.5008827866 & 0.0702507214 & 0.0702566818 & 3.586693173802 \\
$\vdots$ & $\vdots$ & $\vdots$ & $\vdots$ & $\vdots$ & $\vdots$ & $\vdots$ \\
$\infty$ & $\infty$ & 0.5009573929 & 0.5009566265 & 0.0702500491 & 0.0702525913 & 3.586693173783 \\
\hline
\end{tabular}

TABLE 2. Points $\left(x_{n}, y_{n}, \lambda_{n}\right)$ on branches of period $2^{n}$ at which there is a zero eigenvalue. The point $\left(x_{n}^{*}, y_{n}^{*}\right)$ is half way round the periodic orbit from the point $\left(x_{n}, y_{n}\right)$.

\begin{tabular}{|c|c|r|r|}
\hline$n$ & $\kappa_{n}$ & \multicolumn{1}{|c|}{$\gamma_{n}$} & \multicolumn{1}{c|}{$\eta_{n}$} \\
\hline 2 & - & -3.520240 & -2.800035 \\
3 & -7.243107 & 5.989284 & -10.193096 \\
4 & -10.969658 & -1.187539 & -49.177923 \\
5 & -8.567199 & -2.249368 & 0.703237 \\
6 & -9.398249 & -2.545391 & -3.593644 \\
7 & -9.149517 & -2.581882 & -3.750937 \\
8 & -9.117912 & -2.526007 & -4.629019 \\
9 & -9.252171 & -2.555352 & -4.326671 \\
10 & -9.104346 & -2.491145 & -4.683739 \\
\hline
\end{tabular}

TABLE 3. Estimates of the parameter and spatial scaling constants.

case, then the eigenvalues of $G_{X}(0)$ are zero and $g_{y}(0,0)$, and since we have only one zero eigenvalue at each of the singular points, we also assume that $g_{y}(0,0) \neq 0$. With these assumptions, we can say more about the solutions of the fixed point equation (10).

Lemma 3.3. Suppose that the solution $G(X)=(f(x, y), g(x, y))^{T}$ of (10) satisfies $f(x, y)=$ $\tilde{f}(x)$, with $\tilde{f}^{\prime}(0)=0$, and $g_{y}(0,0) \neq 0$. Then $g_{y}(1,1)=1$ and either $\gamma=\eta$ or $g_{x}(0,0)=0$.

Proof. Since $f_{x}(0,0)=\tilde{f}^{\prime}(0)=0$ and $f_{y}(x, y)=0$ for all $x, y$, we can rewrite (11) as

$$
\begin{aligned}
\left(\begin{array}{cc}
0 & 0 \\
g_{x}(0,0) & g_{y}(0,0)
\end{array}\right) & =\left(\begin{array}{ll}
\gamma & 0 \\
0 & \eta
\end{array}\right)\left(\begin{array}{cc}
\tilde{f}^{\prime}(1) & 0 \\
g_{x}(1,1) & g_{y}(1,1)
\end{array}\right) \\
& \left(\begin{array}{cc}
0 & 0 \\
g_{x}(0,0) & g_{y}(0,0)
\end{array}\right)\left(\begin{array}{cc}
\frac{1}{\gamma} & 0 \\
0 & \frac{1}{\eta}
\end{array}\right) \\
& =\left(\begin{array}{cc}
0 & 0 \\
\frac{\eta}{\gamma} g_{y}(1,1) g_{x}(0,0) & g_{y}(1,1) g_{y}(0,0)
\end{array}\right) .
\end{aligned}
$$

The conclusions of the Lemma follow from solving the two equations obtained from the bottom row of this matrix equation. 
In the case when $f(x, y)=\tilde{f}(x)$, we find that the first equation of (10) decouples from the second and it reduces to

$$
\tilde{f}(x)=\gamma \tilde{f}\left(\tilde{f}\left(\frac{x}{\gamma}\right)\right)
$$

which is essentially the same as the fixed point equation (5) derived from the onedimensional renormalisation theory. Thus, the solution of this equation is given by $\gamma=-\alpha$ (as we conjectured earlier) and $\tilde{f}(x)=f_{F}(x)$.

It then remains to find the function $g(x, y)$ and the scaling parameter $\eta$, which can be found by solving the equations

$$
g(x, y)=\eta g\left(f_{F}\left(-\frac{x}{\alpha}\right), g\left(-\frac{x}{\alpha}, \frac{y}{\eta}\right)\right), \quad g(0,0)=1 .
$$

We note that Lemma 3.3 suggests that there may be two solutions of this equation, and this is indeed the case.

Theorem 3.4. There are two solutions of the renormalisation problem (12), which are

(i) $g(x, y)=y-x+f_{F}(x), \eta=-\alpha$;

(ii) $g(x, y)=y+h(x)$ where $h(x)$ and $\eta$ are the solution of the equations

$$
h=\mathcal{L} h, \quad h(0)=1,
$$

where

$$
\mathcal{L} h(x)=\eta\left[h\left(-\frac{x}{\alpha}\right)+h\left(f_{F}\left(-\frac{x}{\alpha}\right)\right)\right] .
$$

The solution of these equations is found numerically to be $\eta=-4.58619671 \ldots$ and

$$
h(x)=1-2.441004 x^{2}+0.098680 x^{4}+0.144460 x^{6}+O\left(x^{8}\right)
$$

Proof.

(i) Substituting the given function $g(x, y)$ and $\eta=-\alpha$ into the right hand side of (12), and using the fact that $f_{F}$ is a fixed point of the operator $T$ given by (4), it is easily verified that it simplifies to the left hand side. Also $g(0,0)=f_{F}(0)=1$ and so the normalisation condition is satisfied as well.

(ii) Substituting the given function $g(x, y)$ into (12), it easily simplifies to (13).

We note that both of these solutions satisfy $g_{y}(1,1)=1$, as required by Lemma 3.3. Also, the first solution has $\gamma=\eta$ and the second solution has $g_{x}(0,0)=0$ since it is an even function of $x$. Thus, these two solutions match the two types of solutions described in Lemma 3.3.

The second solution has been found previously by Kuznetsov et al. [23] in a study of two-dimensional maps with uni-directional coupling. They then go on to describe a period-doubling bifurcation in the renormalisation equation which occurs when the degree of the function at the maximum point is varied continuously, which is not relevant to this work.

We also note that the scaling parameter $\eta$ for the second solution is close to the estimated value of -4.68 which we found from our example. Thus, we assume that this second solution is the one that corresponds to the limit of the renormalisation process for the alternating period-doubling cascade. It remains to find the largest eigenvalue in magnitude of the linearisation of the renormalisation operator evaluated at the fixed point solution, since this should correspond to the parameter scaling constant $\kappa$.

To find the parameter scaling associated to the renormalisation operator we must examine the eigenvalues and associated eigenfunctions of the linearisation of the renormalisation 
operator. Writing a small perturbation from the fixed point $(f, g)=\left(f_{F}(x), y+h(x)\right)$ of the renormalisation operator $S$ as $\Psi(x, y)=\left(\psi_{1}(x, y), \psi_{2}(x, y)\right)^{T}$, we find that the linearisation evaluated at the fixed point $L$ is given by

$$
L \Psi=\left(\begin{array}{cc}
L_{1,1} & 0 \\
L_{2,1} & L_{2,2}
\end{array}\right)\left(\begin{array}{l}
\psi_{1} \\
\psi_{2}
\end{array}\right)
$$

where

$$
\begin{aligned}
& L_{1,1} \psi_{1}(x, y)=\gamma f_{F}^{\prime}\left(f_{F}\left(\frac{x}{\gamma}\right)\right) \psi_{1}\left(\frac{x}{\gamma}, \frac{y}{\eta}\right)+\gamma \psi_{1}\left(f_{F}\left(\frac{x}{\gamma}\right), \frac{y}{\eta}+h\left(\frac{x}{\gamma}\right)\right), \\
& L_{2,1} \psi_{1}(x, y)=\eta h^{\prime}\left(f_{F}\left(\frac{x}{\gamma}\right)\right) \psi_{1}\left(\frac{x}{\gamma}, \frac{y}{\eta}\right), \\
& L_{2,2} \psi_{2}(x, y)=\eta \psi_{2}\left(\frac{x}{\gamma}, \frac{y}{\eta}\right)+\eta \psi_{2}\left(f_{F}\left(\frac{x}{\gamma}\right), \frac{y}{\eta}+h\left(\frac{x}{\gamma}\right)\right) .
\end{aligned}
$$

Since the linearisation has this lower triangular structure its eigenvalues will either be eigenvalues of the operator $L_{1,1}$ or eigenvalues of the operator $L_{2,2}$.

If $L_{1,1}$ has an eigenvalue $\tau$, then the associated eigenfunction will be of the form $\Psi(x, y)=\left(\psi_{1}(x, y), \psi_{2}(x, y)\right)^{T}$, where $\psi_{1}(x, y)$ is an eigenfunction of the reduced problem $L_{1,1} \psi_{1}=\tau \psi_{1}$ and $\psi_{2}$ is found by solving

$$
\left(L_{2,2}-\tau I\right) \psi_{2}=-L_{2,1} \psi_{1},
$$

which will have a unique solution provided that $\tau$ is not also an eigenvalue of $L_{2,2}$ and $-L_{2,1} \psi_{1}$ is in the range of $L_{2,2}-\tau I$.

If $L_{2,2}$ has an eigenvalue of $\tau$, then the associated eigenfunction will be of the form $\Psi(x, y)=\left(0, \psi_{2}(x, y)\right)^{T}$, where $\psi_{2}(x, y)$ is an eigenfunction of the reduced problem $L_{2,2} \psi_{2}=$ $\tau \psi_{2}$. We also note that the eigenfunctions do not have to retain the structure of the fixed point, for which $f$ is a function only of $x$ and $g$ consists of $y$ plus a function of $x$. Thus, we must consider the possibility of $\Psi$ being a function of both $x$ and $y$ in both its components.

Solving the renormalisation problem numerically in two space dimensions, and then finding the eigenvalues of the linearisation evaluated at the fixed point, we find that there are two eigenvalues at +1 and six eigenvalues that are greater than one in modulus, which are given by

$$
\begin{aligned}
& \tau_{1}=4.66920161, \quad \tau_{2}=-2.50290788, \quad \tau_{3}=-9.17239342, \\
& \tau_{4}=1.83234739, \quad \tau_{5}=2.00000000, \quad \tau_{6}=-1.01809854 \text {. }
\end{aligned}
$$

The two eigenvalues at +1 arise since, as discussed above, any fixed point solution of the renormalisation equation (8) generates a two parameter family of such fixed points. The eigenfunctions associated with the +1 eigenvalues are given by the tangent vector to the one-parameter family generated by each of the scaling parameters. Thus, to find these eigenfunctions, we note that

$$
\left.\frac{d}{d \kappa} \kappa f\left(\frac{x}{\kappa}, \frac{y}{\theta}\right)\right|_{\kappa=\theta=1}=f(x, y)-x f_{x}(x, y) .
$$

Substituting the solution $f(x, y)=f_{F}(x)$ gives

Similarly,

$$
\left.\frac{d}{d \kappa} \kappa f\left(\frac{x}{\kappa}, \frac{y}{\theta}\right)\right|_{\kappa=\theta=1}=f_{F}(x)-x f_{F}^{\prime}(x) .
$$

$$
\left.\frac{d}{d \kappa} \theta g\left(\frac{x}{\kappa}, \frac{y}{\theta}\right)\right|_{\kappa=\theta=1}=-x h^{\prime}(x),
$$


and so the eigenfunction associated to one of the eigenvalues at +1 is

$$
\Psi(x, y)=\left(f_{F}(x)-x f_{F}^{\prime}(x),-x h^{\prime}(x)\right)^{T} .
$$

Since the first component of this eigenfunction is non-zero, we know that this is an eigenvalue of the operator $L_{1,1}$. Similarly, for the second scaling parameter $\theta$, we have

$$
\left.\frac{d}{d \theta} \kappa f\left(\frac{x}{\kappa}, \frac{y}{\theta}\right)\right|_{\kappa=\theta=1}=0
$$

since $f(x, y)=f_{F}(x)$ is independent of $y$, and

$$
\left.\frac{d}{d \theta} \theta g\left(\frac{x}{\kappa}, \frac{y}{\theta}\right)\right|_{\kappa=\theta=1}=h(x),
$$

and so the eigenfunction associated to the second eigenvalue at +1 is

$$
\Psi(x, y)=(0, h(x))^{T} .
$$

Clearly, this is an eigenvalue of $L_{2,2}$ since the first component of the eigenfunction is zero.

We now consider more closely the six eigenvalues listed above whose modulus is greater than one and the corresponding eigenfunctions. Since we do not have an analytic expression for the function $h(x)$, we cannot provide analytic expressions for the eigenfunctions in every case. In those cases where we cannot provide an analytic expression for the eigenfunction, we provide a numerical approximation to it instead.

Since the first of our fixed point equations decouples from the second and is the standard one-dimensional renormalisation equation, the linearisation $L_{1,1}$ will have eigenvalues which are known from the one-dimensional problem. In particular, there are two eigenvalues that have modulus greater than one, which are $\delta$ and $-\alpha$ [4]. We will call the eigenfunctions corresponding to these eigenvalues $\psi_{\delta}(x)$ and $\psi_{\alpha}(x)$ respectively and we recall that $\psi_{\alpha}(x)=f_{F}^{\prime}(x)-1$. We note that the eigenvalue $-\alpha$ arises from the coordinate change $x \rightarrow x+\epsilon[9]$.

We now consider these two eigenvalues in the context of the two-dimensional problem.

- $\tau_{1}=\delta$

For this eigenvalue, we have already seen that $\psi_{1}(x, y)=\psi_{\delta}(x)$. The second component of the eigenfunction is $\psi_{2}(x)=\tilde{\psi}_{\delta}(x)$ where $\tilde{\psi}_{\delta}(x)$ must satisfy

$$
L_{2,1} \psi_{\delta}+\left(L_{2,2}-\delta I\right) \tilde{\psi}_{\delta}=0 .
$$

The linear operator $L_{2,2}-\delta I$ is injective since $\delta$ is not found (numerically) to be an eigenvalue of $L_{2,2}$ and so this equation will have a unique solution provided that $L_{2,1} \psi_{\delta}$ is in the range of $L_{2,2}-\delta I$. Solving this equation numerically gives

$$
\tilde{\psi}_{\delta}(x)=1.55534859-0.70057177 x^{2}-0.29201524 x^{4}+0.08494920 x^{6}+O\left(x^{8}\right) \text {. }
$$

- $\tau_{2}=-\alpha$

It is known [9] that this eigenvalue arises due to the coordinate change $x \rightarrow x+\epsilon$ and, for this problem, has the corresponding eigenfunction

$$
\Psi(x)=\left(f_{F}^{\prime}(x)-1, h^{\prime}(x)\right)^{T} .
$$

In order to find eigenvalues of $L_{2,2}$, we could initially make the assumption that $\psi_{2}$ is a function only of $x$. In this case, the eigenvalues and corresponding eigenfunctions can be found from the one-dimensional fixed point problem (13). The eigenvalues with modulus greater than one in this case are $\tau_{3}$ and $\tau_{4}$, and so we now consider these eigenvalues in more detail. Since these are eigenvalues of $L_{2,2}$ we know that $\psi_{1}=0$ and so we have only to find $\psi_{2}$. 
- $\tau_{3}=2 \eta=-9.17239342$

Setting $\psi_{2}(x)=1$ gives this eigenvalue.

- $\tau_{4}=-\eta / \alpha=1.83234739$

It is also known [9] that this eigenvalue arises due to the coordinate change $y \rightarrow$ $y+\epsilon x$ which, for this problem, has the corresponding eigenfunction

$$
\Psi=\left(0, f_{F}(x)-x\right)^{T} .
$$

Finally, we must solve the full two-dimensional problem to find any eigenvalues with eigenfunctions which depend on both $x$ and $y$. In this case, we find all four of the above eigenvalues, together with two further eigenvalues, as follows.

- $\tau_{5}=2$

The numerical results suggest that $\tau=2$ is an eigenvalue where the corresponding eigenfunction has the form

$$
\psi_{1}(x, y)=0, \quad \psi_{2}(x, y)=y+\hat{\psi}_{2}(x) .
$$

Since $\psi_{1}=0$, then clearly this is an eigenvalue of $L_{2,2}$. To prove the existence of this eigenvalue, we substitute $\psi_{2}(x, y)$ given in (15) into the eigenvalue problem $L_{2,2} \psi_{2}=\tau \psi_{2}$ which gives

$$
2 y+\mathcal{L} \hat{\psi}_{2}+\eta h\left(-\frac{x}{\alpha}\right)=\tau\left(y+\hat{\psi}_{2}(x)\right)
$$

where $\mathcal{L}$ is defined in (14). Equating the $y$ terms on both sides gives $\tau=2$, as anticipated, and the function $\hat{\psi}_{2}$ is then found by solving the equation

$$
(\mathcal{L}-2 I) \hat{\psi}_{2}(x)=-\eta h\left(-\frac{x}{\alpha}\right) .
$$

Since $\hat{\psi}_{2}$ is a function only of $x$, then $\mathcal{L} \hat{\psi}_{2}=L_{2,2} \hat{\psi}_{2}$ and we have found (numerically) that $L_{2,2}$ operating on functions of $x$ does not have an eigenvalue $\tau=2$. Thus, $\mathcal{L}-2 I$ is injective on the space of functions of $x$ and so this equation will have a unique solution provided that the right hand side function is in the range of the linear operator. The numerical solution of this equation is

$\hat{\psi}_{2}(x)=-1.17774979+2.03186048 x^{2}-0.13554484 x^{4}-0.03113801 x^{6}+O\left(x^{8}\right)$.

- $\tau_{6}=\delta / \eta=-1.01809854$

The numerical results indicate that $\tau=\delta / \eta$ is an eigenvalue and that the corresponding eigenfunction has the form

$$
\psi_{1}(x, y)=\psi_{1,1}(x)+y \psi_{1,2}(x), \quad \psi_{2}(x, y)=\psi_{2,1}(x)+y \psi_{2,2}(x) .
$$

To prove the existence of this eigenvalue, we substitute the template functions given in (16) into the eigenvalue problem $L \Psi=\tau \Psi$. Equating the terms that involve $y$ and those that do not in both equations gives the four equations

$$
\begin{aligned}
L_{1,1} \psi_{1,1}+\gamma h\left(\frac{x}{\gamma}\right) \psi_{1,2}\left(f_{F}\left(\frac{x}{\gamma}\right)\right) & =\tau \psi_{1,1} \\
\frac{1}{\eta} L_{1,1} \psi_{1,2} & =\tau \psi_{1,2} \\
L_{2,1} \psi_{1,1}+L_{2,2} \psi_{2,1}+\eta h\left(\frac{x}{\gamma}\right) \psi_{2,2}\left(f_{F}\left(\frac{x}{\gamma}\right)\right) & =\tau \psi_{2,1} \\
\frac{1}{\eta}\left(L_{2,1} \psi_{1,2}+L_{2,2} \psi_{2,2}\right) & =\tau \psi_{2,2}
\end{aligned}
$$

Now $L_{1,1}=J_{f_{F}}$ when acting on functions of $x$, and we know that this linear operator has eigenvalues $-\alpha$ and $\delta$. If $\sigma$ is an eigenvalue of $L_{1,1}$, then clearly from 
(18), $\tau=\sigma / \eta$ is an eigenvalue of $L$. Therefore, $\tau=-\alpha / \eta$ and $\tau=\delta / \eta$ are both eigenvalues. However, $|-\alpha / \eta|<1$ and so is of no interest. The other eigenvalue $\tau=\delta / \eta$ is slightly greater than one in modulus and this is the eigenvalue we are now considering.

The solution of equations (18) and (20) is given by

$$
\psi_{1,2}=\psi_{\delta}, \quad \psi_{2,2}=\tilde{\psi}_{\delta}
$$

Now $\tau=\delta / \eta$ is not found (numerically) to be an eigenvalue of either $L_{1,1}$ or $L_{2,2}$ when restricted to functions of $x$, and so the linear operators $L_{1,1}-(\delta / \eta) I$ and $L_{2,2}-(\delta / \eta) I$ are injective. If the remaining terms are in the range, then equations (17) and (19) will have a unique solution. Numerically, $\psi_{1,1}$ and $\psi_{2,1}$ are found to be

$$
\begin{aligned}
& \psi_{1,1}(x)=0.32215488+0.13336107 x^{2}-0.18179046 x^{4}+0.02654470 x^{6}+O\left(x^{8}\right) \\
& \psi_{2,1}(x)=-0.57631257+3.52736562 x^{2}-2.23557075 x^{4}+0.28272664 x^{6}+O\left(x^{8}\right)
\end{aligned}
$$

Since $\psi_{1} \neq 0$ then clearly this is an eigenvalue of $L_{1,1}$.

This solution to the renormalisation problem has been found previously by Kuznetsov et al. [23] as well as the eigenvalues of the linearisation. Our solution and eigenvalues agree with theirs, but most of our eigenfunctions are different from theirs, not just by a scaling factor, (many of their eigenfunctions do not include a constant term, which all of ours do) which we are unable to explain.

To summarise, we note that the linear operators $L_{1,1}$ and $L_{2,2}$ each have three unstable eigenvalues, one arising from a coordinate change, one associated with a change in the structure of the solutions, plus one other. We are not interested in the two eigenvalues associated with a coordinate change $\left(\tau_{2}, \tau_{4}\right)$. Since the alternating period-doubling cascade can be found using only two parameters, it seems that the two eigenvalues with eigenfunctions that break the structure of the solution $\left(\tau_{5}, \tau_{6}\right)$ are also not relevant. This leaves us with two unstable eigenvalues $\left(\tau_{1}=\delta, \tau_{3}=2 \eta\right)$ and hence the fixed point of the renormalisation problem has a two-dimensional unstable manifold.

Standard renormalisation theory then tells us that the parameter values at successive singular points through the cascade, $\lambda_{r}$, converge to the limiting value $\lambda_{\infty}$ as

$$
\lambda_{r}-\lambda_{\infty}=A \delta^{-r}+B(2 \eta)^{-r}+\cdots
$$

Since $|2 \eta|>\delta$, then $(2 \eta)^{-r}$ converges to zero faster than $\delta^{-r}$ and so we would expect generically that

$$
\lambda_{r}-\lambda_{\infty} \approx A \delta^{-r}
$$

since this is the slowest converging of the two terms. Clearly this is what happens with the usual period-doubling cascade.

However, consider the situation when $|A| \ll|B|$. In this case, for moderate values of $r$, we will have

$$
\lambda_{r}-\lambda_{\infty} \approx B(2 \eta)^{-r}
$$

and, since $\eta$ is negative, $\lambda_{r+1}-\lambda_{r}$ will alternate in sign for successive values of $r$. As $r$ increases, the two terms will at some stage become of comparable magnitude and subsequently the first term will dominate, in which case (22) again holds and $\lambda_{r+1}-\lambda_{r}$ will have the same sign. Thus, in this case, we would expect to see that the singular points initially alternate and then proceed in one direction. This is precisely what is observed in a neighbourhood of an alternating period-doubling cascade. Only at a critical value of a second parameter, which corresponds to $A=0$, will we see the singular points alternate for all $r$, which corresponds to an alternating period-doubling cascade. Clearly this corresponds to the special case of convergence to the fixed point of the renormalisation problem along 


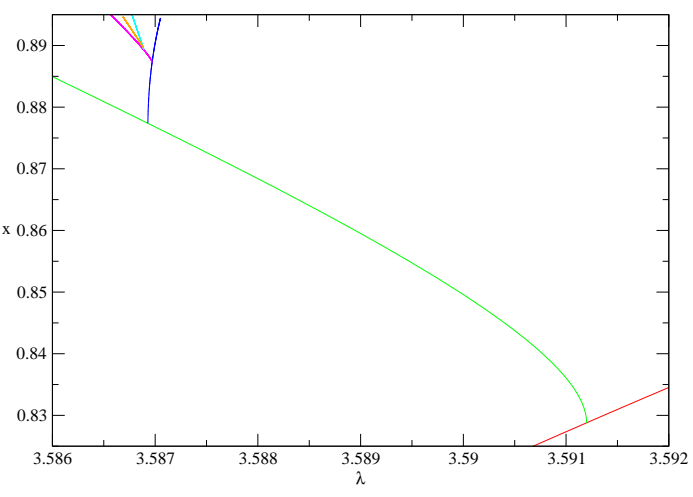

(a) $\mu=0.3$.

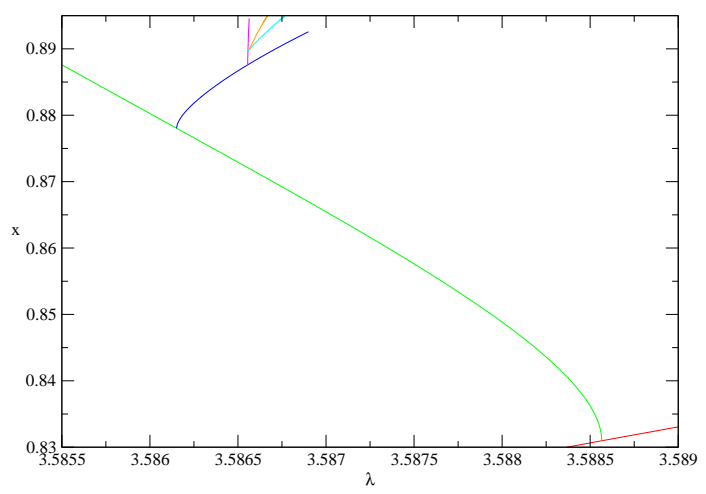

(b) $\mu=0.7$.

Figure 8. Period-doubling cascades for different values of $\mu$. Red: period 2; green: period 4; blue: period 8; magenta: period 16; cyan: period 32; orange: period 64 .

the one-dimensional manifold corresponding to the eigenvalue $\tau_{3}=2 \eta$, rather than along the manifold associated with the eigenvalue $\tau_{1}=\delta$ as in all other cases. Moreover, as the second parameter passes through the critical value for the alternating period-doubling cascade, the coefficient $A$ in (21) will change sign, which is also associated with a change in the direction of convergence of the period-doubling cascade to the limiting value since $A>0$ implies that $\lambda_{r}>\lambda_{\infty}$ and $A<0$ gives $\lambda_{r}<\lambda_{\infty}$. This unfolding of an alternating period-doubling cascade is studied in more detail elsewhere $[15,16]$.

We now compare these theoretical results with our example.

Example 3.5. To compare these theoretical results with our earlier example, we first redefine the function $F_{1}$ to include a second parameter as

$$
F_{1}\left(x_{n}, y_{n}, \lambda, \mu\right)=\lambda x_{n}\left(1-x_{n}\right)-y_{n}^{2}\left(\mu+1.7 x_{n}\right)
$$

Previously, we considered the case of $\mu=0.5050541762$ at which the alternating perioddoubling cascade occurs. The period-doubling cascades for $\mu=0.3$ and $\mu=0.7$, either side of the alternating cascade, are shown in Fig. 8. Both cascades start with a forward period-doubling bifurcation on the period 1 branch. The first bifurcation shown is the next one on the period 2 branch, which is clearly a backward bifurcation. Thus, we see that at $\mu=0.3$, there is initially an alternating cascade which is followed by a backward cascade, whereas at $\mu=0.7$, the initial alternating cascade is followed by a forward cascade, as predicted from the renormalisation theory.

The eigenvalue $\tau_{3}=2 \eta=-9.17239342$, which we have shown above gives the parameter scaling for an alternating period-doubling cascade, agrees well with the last two parameter scaling estimates given by $\kappa_{9}=-9.252171$ and $\kappa_{10}=-9.104346$ listed in Table 3 . There is also good agreement for the two spatial scaling constants. The approximate values obtained from our example are $\gamma_{10}=-2.491145$ and $\eta_{10}=-4.683739$ and these are close to the theoretical values of $\gamma=-\alpha=-2.50290788$ and $\eta=-4.58619671$.

Thus, we have excellent agreement between the scalings found numerically and one solution of the two-dimensional renormalisation problem.

\section{Conclusions}

We have considered in detail an alternating period-doubling cascade, in which successive period-doubling bifurcations branch in opposite directions, which occurs only for a critical value of a second parameter. We have shown that for two-dimensional maps, there are 
two possible different cases for the movement of the eigenvalues through such a cascade. We have studied one of these, with the assumption that there is a singular point on every branch. The limiting behaviour of such a cascade has been considered in terms of solutions of a two-dimensional renormalisation problem, and excellent agreement between the theory and a numerical example has been obtained. We have also described how the alternating period-doubling cascade is the transition point at which a period-doubling cascade changes direction in the limit.

For a standard period-doubling cascade, the limiting value $\lambda_{\infty}$ is often referred to as the chaos boundary, which is certainly the case for the logistic map, as well as many others. However, for an alternating period-doubling cascade, we note that the limiting value for a type I cascade is unlikely to be the point of transition to chaos, since there are infinitely many stable periodic solutions at that point.

There are interesting parallels between the period-doubling cascades that we have studied, and the behaviour of a superball bouncing on a flat surface! It has been found that the superball may have several direction reversals as it bounces, before eventually bouncing in only one direction, except for one critical parameter value at which it alternates in direction at every bounce [24].

This work could be extended by considering the case when there is only a singular point on every other branch, rather than on every branch as we have assumed, in a type I cascade. A type II cascade could also be considered.

\section{REFERENCES}

[1] Y.A. Kuznetsov. Elements of Applied Bifurcation Theory, second edition, Springer, Berlin, 2004.

[2] R. May. Simple mathematical models with very complicated dynamics, Nature 261, 459-467, 1976.

[3] M.J. Feigenbaum. Quantitative universality for a class of nonlinear transformations, J. Stat. Phys. 19, 25-52, 1978.

[4] M.J. Feigenbaum. The universal metric properties of nonlinear transformations, J. Stat. Phys. 21, 669-706, 1979.

[5] M.J. Feigenbaum. Universal behavior in nonlinear systems, Los Alamos Sci. 1, 4-27, 1980.

[6] M. Hénon. A two-dimensional mapping with a strange attractor, Comm. Math. Phys. 50, 69-77, 1976.

[7] P. Collet, J.P. Eckmann and H. Koch. Period doubling bifurcations for families of maps on $\mathbb{R}^{n}, J$. Stat. Phys. 25, 1-14, 1981.

[8] C. Sparrow. The Lorenz equations: bifurcations, chaos, and strange attractors, Volume 41, Applied Mathematical Sciences, Springer-Verlag, 1982.

[9] J.M. Greene, R.S MacKay, F. Vivaldi and M.J. Feigenbaum. Universal behaviour in families of area-preserving maps, Physica D 3, 468-486, 1981.

[10] R.S. MacKay. Renormalisation in Area-Preserving Maps, Volume 6, Advanced Series in Nonlinear Dynamics, World Scientific, 1993.

[11] S.Y. Kim. Period p-tuplings in coupled maps, Phys. Rev. E Stat. Phys. Plasmas Fluids Relat. Interdiscip. Topics 54, 3393-3418, 1996.

[12] A.P. Kuznetsov, S.P. Kuznetsov and I.R. Sataev. A variety of period-doubling universality classes in multi-parameter analysis of transition to chaos, Physica D 109, 91-112, 1997.

[13] S.P. Kuznetsov, A.P. Kuznetsov and I.R. Sataev. Multiparameter critical situations, universality and scaling in two-dimensional period-doubling maps, J. Stat. Phys. 121, 697-748, 2005.

[14] E. Doedel, A.R. Champneys, T.F. Fairgrieve, Y.A. Kuznetsov, B. Sandstede and X. Wang. AUTO97: Continuation and bifurcation software for ordinary differential equations (with HOMCONT), Technical report, Concordia University, 1997.

[15] N. Bristow. Mode Interactions and Transitions Associated to Period-Doublings in Maps with Two Parameters, PhD thesis, University of Surrey, 2010.

[16] P.J. Aston and N. Bristow. A two-parameter analysis of the direction reversal of a period-doubling cascade for two-dimensional maps. In preparation.

[17] K.M. Briggs. A precise calculation of the Feigenbaum constants, Math. Comp. 57, 435-439, 1991. 
[18] J.P. Eckmann, H. Koch and P. Wittwer. Existence of a fixed point of the doubling transformation for area-preserving maps of the plane, Phys. Rev. A 26, 720-722, 1982.

[19] S.P. Kuznetsov and I.R. Sataev. A new type of period-doubling scaling behavior in two-dimensional area-preserving map, Physics Letters A, 350, 110-116, 2006.

[20] A.S. Ivanova, S.P. Kuznetsov and A.H. Osbaldestin. Universality and scaling in networks of perioddoubling maps with a pacemaker Discrete Dynamics in Nature and Society, 1-19, 2006.

[21] J.P. Boyd. Chebyshev and Fourier Spectral Methods, second edition, Dover, New York, 2001.

[22] C. Canuto, M.Hussaini, A.Quarteroni and T.Zang. Spectral Methods in Fluid Dynamics, Springer Verlag (Berlin and New York), 1988.

[23] S.P. Kuznetsov, A.A. Mailybaev and I.R. Sataev. Birth of a new class of period-doubling scaling behavior as a result of bifurcation in the renormalization equation, J. Stat. Phys. 130, 599-616, 2008.

[24] P.J. Aston. and R. Shail. The dynamics of a bouncing superball with spin, Dyn. Sys. 22, 291-322, 2007.

Department of Mathematics, University of Surrey, Gulldford, Surrey, GU2 7XH 